\title{
Small-scale isolation of synaptic vesicles from mammalian brain
}

\author{
Saheeb Ahmed ${ }^{1,4}$, Matthew Holt ${ }^{1,2,4}$, Dietmar Riedel ${ }^{3}$ \& Reinhard Jahn ${ }^{1}$ \\ ${ }^{1}$ Department of Neurobiology, Max Planck Institute for Biophysical Chemistry, Göttingen, Germany. ${ }^{2}$ Laboratory of Glia Biology, Vlaams Instituut voor Biotechnologie \\ (VIB) Center for the Biology of Disease, Katholieke Universiteit (KU) Leuven, Leuven, Belgium. ${ }^{3}$ Electron Microscopy Group, Max Planck Institute for Biophysical \\ Chemistry, Göttingen, Germany. ${ }^{4}$ These authors contributed equally to this work. Correspondence should be addressed to R.J. (rjahn@gwdg.de).
}

Published online 25 April 2013; doi:10.1038/nprot.2013.053

\begin{abstract}
Synaptic vesicles (SVs) are essential organelles that participate in the release of neurotransmitters from a neuron. Biochemical analysis of purified SVs was instrumental in the identification of proteins involved in exocytotic membrane fusion and neurotransmitter uptake. Although numerous protocols have been published detailing the isolation of SVs from the brain, those that give the highest-purity vesicles often have low yields. Here we describe a protocol for the small-scale isolation of SVs from mouse and rat brain. The procedure relies on standard fractionation techniques, including differential centrifugation, rate-zonal centrifugation and size-exclusion chromatography, but it has been optimized for minimal vesicle loss while maintaining a high degree of purity. The protocol can be completed in less than $1 \mathrm{~d}$ and allows the recovery of $\sim 150 \mu \mathrm{g}$ of vesicle protein from a single mouse brain, thus allowing vesicle isolation from transgenic mice.
\end{abstract}

\section{INTRODUCTION}

SVs are essential organelles found in the presynaptic terminal that act as a store of neurotransmitters. After $\mathrm{Ca}^{2}+$ influx into the presynaptic terminal during an action potential, SVs fuse with the plasma membrane (exocytosis), resulting in the release of neurotransmitters. After exocytosis, the SV membrane is recovered by endocytosis and used to reform vesicles, which are then refilled with neurotransmitters and used for subsequent rounds of exocytosis. Thus, understanding the composition of these vesicles and how this influences their trafficking within the presynaptic terminal is essential to understanding neurotransmitter release.

Protocols for isolating SV from brain tissue were originally developed in the 1960s. Biochemical characterization of these early preparations was instrumental in proving that SVs are storage organelles for neurotransmitters, although many of the trafficking proteins known to be instrumental in membrane docking and fusion were also first identified in purified SVs ${ }^{1}$. In fact, interest in the biochemical characterization of the SV continues unabated, as documented by several recent studies addressing the SV proteome ${ }^{2,3}$. Furthermore, numerous transgenic mouse lines, with defined changes in presynaptic protein expression, are now available (http://www.jax.org/), indicating that reliable protocols are needed to allow the high-yield and high-purity isolation of vesicles from small amounts of starting material. Here we describe the process for obtaining such a preparation.

\section{Methods for isolating SVs}

With an average diameter of $40-50 \mathrm{~nm}$, SVs are among the smallest trafficking organelles in mammalian cells. In addition, they are highly abundant in brain tissue, with $~ 5 \%$ of the total protein content of the mammalian central nervous system being contributed by SV proteins. It is these two remarkable properties that allow the application of standard subcellular fractionation techniques to SV purification. After the first description of a protocol for the isolation of vesicles from brain extracts ${ }^{4,5}$, a large number of procedures were developed (which are comprehensively reviewed by Whittaker ${ }^{6}$ and summarized in Fig. 1).

The conventional methods for isolation of SVs can be divided into two main groups. The first involves the intermediate isolation of isolated nerve terminals (synaptosomes), which are generated during gentle homogenization of brain tissue ${ }^{7}$.SVs are then released by hypotonic rupture of synaptosomes and are further purified by differential and density-gradient centrifugation. The advantage of this procedure is that synaptosomes are comparatively large particles that sediment at low $g$-forces, whereas smaller particles (such as ribosomes), membrane fragments or small vesicles from other sources all remain in the supernatant. After osmotic lysis, larger particles (including plasma membranes, mitochondria and myelin) are removed by differential centrifugation, leaving a supernatant highly enriched in SVs. The disadvantage of this procedure is that during initial homogenization many nerve terminals are damaged and release their SVs (at least partially) into the supernatant, resulting in a major loss of vesicles and thus low final yields.

The second group of protocols involves direct isolation of SVs from brain homogenates. Accordingly, homogenization conditions can be harsher, and protocols using shock-frozen brains have even been described ${ }^{8}$. These protocols usually allow for higher yields, but it is more difficult to remove contaminating membranes and particles, normally resulting in lower purity. To obtain higher purity, further loss occurs during additional purification that cancels out any initial improvements in the yield.

In both cases, further purification usually involves a combination of differential centrifugation and density-gradient centrifugation. Differential centrifugation takes advantage of the fact that vesicles are among the smallest organelles known, with high-speed ultracentrifugation being required to pellet them. Therefore, SVcontaining samples can be precentrifuged at relatively high $g$-forces, pelleting the larger membranes, leaving the SVs free in the supernatant. Density gradients are subsequently carried out as rate-zonal centrifugations, in which SVs migrate as a band that can be collected after it has separated away from contaminants. Alternatively, it is possible to enrich SVs by isopycnic density-gradient centrifugation; however, because of the small size of the vesicles, very long centrifugation times are required before density equilibrium is reached. Owing to the small and homogenous size of SVs, sizeexclusion chromatography is frequently used as a final purification step to further remove contaminating membranes and (if needed) any remaining cytosolic proteins. 
Synaptosomal methods:

Mild initial homogenization

Low-speed >

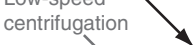

Increasing Synaptosome isolation

speed Low-speed Osmotic lysis of synaptosomes

centrifugation Release of SVs

Clearing of heavy membranes

e.g., plasma membrane

from SVs

Low-speed

centrifugation

Direct release of SVs

Complete disruption of neurons

Direct release methods:

Harsh initial homogenization

Figure 1 | Flow schematic illustrating the basic steps in SV purification using classical size-fractionation techniques.

\section{Experimental design}

Our recently established procedure allows the isolation of functionally active SVs from mammalian (mouse and rat) brain ${ }^{9}$. The protocol is essentially a hybrid of existing techniques, which have been optimized for yield and purity and which can be carried out in $24 \mathrm{~h}$ or less. The protocol proceeds via the initial mild homogenization of the brain to reduce fragmentation and vesiculation of large membranes, and it thus involves the isolation of a synaptosomeenriched fraction from which SVs are released by osmotic rupture. Contrary to most other protocols, however, the vesicles released during the initial homogenization are also collected and combined with those released from the synaptosomes for further purification. Subsequent purification steps include a simplified rate-zonal sucrose density-step gradient, followed by size-exclusion chromatography on Sephacryl S-1000. With respect to purity, the final fraction compares well with the classical procedure originally developed by Nagy et al. ${ }^{10}$ (and used with minor variations ${ }^{3,11}$ ), which currently constitutes the 'gold standard' in SV purification, despite being much more elaborate than the method described here. Importantly, though, the yield from our method is approximately sixfold higher. Thus, our protocol can be adopted for the isolation of SVs from a single mouse brain and, by extension, it opens up the possibility of using transgenic mouse lines in vesicle purification.

The protocol allows the quick and easy isolation of SVs from a single adult mouse brain ${ }^{9}$. However, it can easily be scaled up or down by a corresponding increase or reduction in the experimental volumes (noting that, in differential centrifugation, a change in volume will result in a change in the effective sedimentation properties). A flow diagram illustrating the procedure is given in Figure 2a. Illustrative photographs of the various experimental stages can be found in Supplementary Figure 1.

It should also be noted that the preparation of analytical amounts of SVs has been reported, using variations on the purification methods described here. These protocols include the use of floatation gradients, following synaptosome lysis, to purify vesicles ${ }^{12}$ and immunoaffinity purification, which takes advantage of the high binding affinity and specificity of an antibody for its antigen to allow SVs to be rapidly isolated ${ }^{13}$ (particularly after rate-zonal centrifugation of 'semipure' preparations on sucrose gradients ${ }^{2}$ ). For example, our protocol can be conveniently combined with immunoisolation, (i.e., by using our 'combined supernatants' as the starting material). Purity and functionality of vesicle preparations prepared using these variations can be checked using the same methods described in the ANTICIPATED RESULTS section.

\section{MATERIALS}

\section{REAGENTS}

$\triangle$ CRITICAL All reagents are available from Sigma-Aldrich unless otherwise stated and should be of analytical grade or higher.

- Sucrose (cat. no. S7903)

- HEPES (cat. no. H7523)

- Tris-(hydroxymethyl)aminomethane (Tris; cat. no. T6791)

- Protease inhibitors: PMSF (Roth, cat. no. 6367.3) and pepstatin A

(Pepta Nova, cat. no. 4397)

- Sephacryl S-1000 Superfine SF, 300-400-nm exclusion size (GE Healthcare, cat. no. 17-0476-01)

- Marker antibodies (a full list of antibodies and suppliers can be found in

Table 1)

- $\mathrm{ddH}_{2} \mathrm{O}$

- Mice, 6-8 weeks postnatal, 25-30 g body weight, $0.5 \mathrm{~g}$ wet brain weight; or rats ! CAUTION All experiments involving live rodents must conform to all relevant institutional and governmental ethics regulations.

$\Delta$ CRITICAL It should be noted that, in our hands, one whole brain from an adult rat is equivalent to two brains from adult mice on a wet weight: wet weight basis. Although the procedure is described for mouse brains, rat brains can easily be used in this protocol with minor modifications, as indicated below.

\section{EQUIPMENT}

- Sterile filtration unit with a $0.22-\mu \mathrm{m}$ filter (Corning, cat. no. 431097) and vacuum pump

- Motor-driven glass-Teflon homogenizers ( $1 \mathrm{ml}$ and $17 \mathrm{ml}$ with $0.1-0.15-\mathrm{mm}$ clearance) (Omnilab, cat. nos. 5651690 and 5651694, respectively)
- Cooled centrifuge; RC6 Plus (Sorvall) with SS34 rotor and 16-ml flanged polycarbonate (nonsealed) tubes with adapters (Beranek Laborgeraete, cat. nos. 252799 and 22006, respectively)

- Ultracentrifuge (OptimaL-90K; Beckman Coulter) with Ti 50.2 and Ti 70.1 rotors and sealed polycarbonate tubes (cat. nos. 355618 and 355603, respectively)

- Equivalent centrifuges and tubes from other manufacturers can be used I CAUTION Observe all safety precautions for the use of centrifuges.

- Chromatography column $(100 \mathrm{~cm}$ length $\times 1 \mathrm{~cm}$ inner diameter

(Bio-Rad, cat. no. 737-1091)

- Peristaltic pump (Gilson, cat. no. F155001)

- Fraction collector, UV detector unit and chart recorder (GE Healthcare, cat. nos. 18-1177-40, 11-0007-54 and 18-1132-32, respectively)

- Typhoon FLA-7000 biomolecular imager (GE Healthcare, cat. no. 28-9558-09) equipped with appropriate filters or ImageQuant LAS 4000 image reader (GE Healthcare, cat. no. 28-9558-10)

\section{REAGENT SETUP}

Sucrose, 2 M Filter $2 \mathrm{M}$ sucrose through a sterile $0.22-\mu \mathrm{m}$ vacuum unit. The $2 \mathrm{M}$ sucrose should be stored sterile at $4^{\circ} \mathrm{C}$ (for at least 6 months), or divided into aliquots and frozen at $-20^{\circ} \mathrm{C}$ until use (up to 12 months). HEPES, $1 \mathrm{M}$ Prepare two stock solutions with $\mathrm{pH}$ adjusted to $7.4 \mathrm{using}$ $\mathrm{NaOH}$ or KOH. $1 \mathrm{M}$ HEPES can be stored at $4{ }^{\circ} \mathrm{C}$ indefinitely. Homogenization buffer Mix 320 mM sucrose and 4 mM HEPES (pH 7.4, adjusted with $\mathrm{NaOH})$. $\triangle$ CRITICAL This working sucrose solution should be made fresh on the day of use and kept at $4{ }^{\circ} \mathrm{C}$ until use. 
a
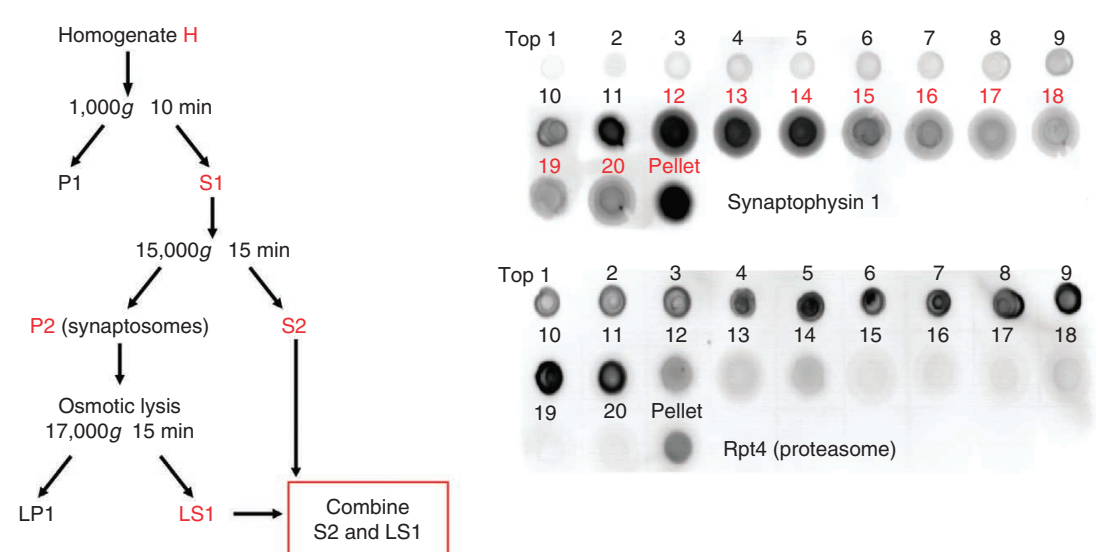

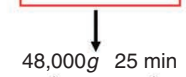

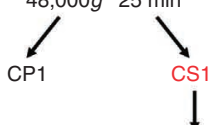

$0.7 \mathrm{M}$ sucrose cushion $133,000 \mathrm{~g} 1 \mathrm{~h}$

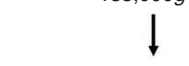

Take $500-\mu l$ fractions from top and dot blot

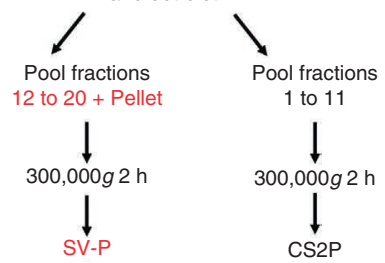

$\downarrow$

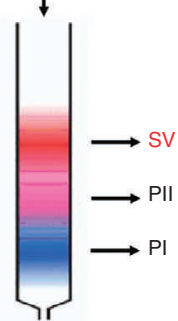

Sephacryl S-1000 (size-exclusion) chromatography
Sucrose cushion Mix $700 \mathrm{mM}$ sucrose and 10 mM HEPES (pH 7.4, adjusted with $\mathrm{KOH}$ ). $\triangle$ CRITICAL This working sucrose solution should be made fresh on the day of use and kept at $4{ }^{\circ} \mathrm{C}$ until use.

Chromatography buffer Mix 100 mM Tris- $\mathrm{HCl}$ (pH 7.6) and $100 \mathrm{mM} \mathrm{KCl}$; filter the mixture through a $0.22-\mu \mathrm{m}$ filter and degas it thoroughly before use. $\triangle$ CRITICAL pH values of Tris buffers are temperature dependent, and thus $\mathrm{pH}$ should be adjusted at the working temperature $\left(4^{\circ} \mathrm{C}\right)$. Buffer can be stored at $4{ }^{\circ} \mathrm{C}$ indefinitely.

PMSF Prepare PMSF as a $200 \mathrm{mM}$ stock solution in $100 \%$ ethanol. When stored at $4{ }^{\circ} \mathrm{C}$, PMSF is stable for a maximum of 6 months. Pepstatin A Prepare pepstatin A as a $1 \mathrm{mg} \mathrm{ml}^{-1}$ stock solution in DMSO. When stored at $4{ }^{\circ} \mathrm{C}$, pepstatin is stable for a maximum of 1 week. Antibodies A full list of all primary antibodies suitable for detecting mouse proteins can be found in Table 1. Most of the listed antibodies should cross-react with the equivalent rat proteins. However, the reader is encouraged to check this for each individual antibody with the listed supplier.
Figure 2 | Schematic of an improved procedure for small-scale isolation of SVs from mammalian brain. (a) A flow diagram illustrating the purification protocol for SVs from mouse brain. Increased yield is achieved by combining SVs released into the supernatant during homogenization (S2) and those released from synaptosomes after osmotic lysis (LS1). Contamination of the SVs is reduced by the use of a sucrose cushion before size-exclusion chromatography on Sephacryl S-1000.

(b) The distribution profiles of synaptophysin (SV marker) and Rpt4 (proteasome marker) after the centrifugation of the CS1 fraction on a sucrose cushion. In the CS1 fraction, SVs are enriched but still heavily contaminated. Direct fractionation of CS1 by size-exclusion chromatography removes some, but not all, of these contaminants (not shown). Prominent among the contaminants are proteasomes, which are effectively removed by use of the sucrose cushion. After centrifugation, fractions are taken and dot blot immunostaining is performed. As can be seen, SVs separate away from proteasomes under appropriate conditions. Further details can be found in Supplementary Figure 2.

For accurate signal quantification from immunoblots, we recommend the use of fluorescently labeled secondary antibodies, which give a wider linear detection range compared with those using the horseradish peroxidaseenhanced chemiluminescence (HRP-ECL) system. We routinely use Cy3 IgG goat anti-mouse and Cy5 IgG goat anti-rabbit (both from Dianova; cat. nos. 115-165-166 and 111-175-144); for ECL detection, we use HRPconjugated goat anti-mouse and HRP-conjugated goat anti-rabbit (both from Bio-Rad, cat. nos. 170-6516 and 170-6515). Secondary antibody stocks are prepared according to the suppliers' instructions. HRP-conjugated antibodies are used at a dilution of 1:2,000 (vol:vol) and Cy3/Cy5 antibodies at a dilution of 1:2,000 (vol:vol). Signals are recorded (and quantified) either using a Typhoon FLA-7000 biomolecular imager equipped with appropriate filters or using an ImageQuant LAS 4000 image reader. We imported the figures in this paper into Photoshop (Adobe), and we adjusted the contrast to best show the levels of proteins in the various subfractions relative to the starting homogenate.

\section{EQUIPMENT SETUP}

$\triangle$ CRITICAL All equipment used during the procedure should be free of detergent. Glassware can be cleaned free of detergent by extensive washing in ethanol and $\mathrm{ddH}_{2} \mathrm{O}$ before use. When large numbers of preparations are required, we recommend purchasing separate equipment.

Sephacryl size-exclusion column Pack the Sephacryl size-exclusion column according to standard instructions, which are available at http://www. gelifesciences.com/webapp/wcs/stores/servlet/catalog/en/GELifeSciences-DE/ products/AlternativeProductStructure_17395/17047601. A $100 \mathrm{~cm} \times 1 \mathrm{~cm}$ column requires $\sim 70 \mathrm{ml}$ of packed resin. Before running the samples, thoroughly equilibrate the column with ten column-volumes of column buffer. To avoid the accumulation of debris, filter all buffers before use. During repetitive runs, we noticed that contaminants accumulate. In this case, we recommend replacing the entire column with new material. Alternatively, the column can be cleaned according to the manufacturer's instructions; simple cleaning with a one-fifth column-volume of $0.1 \mathrm{M} \mathrm{NaOH}$ followed by a onefifth column-volume of $1 \mathrm{M}$ acetic acid should suffice for all but the heaviest contamination. Before further use, thoroughly equilibrate the column in column buffer. The column should not run dry. If this occurs, a new column should be prepared. For long-term storage, equilibrate the column in column buffer containing $0.02 \%$ (wt/vol) sodium azide to prevent microbial growth. 
TABLE 1 | Antibodies used in this study.

\begin{tabular}{|c|c|c|c|c|}
\hline Clathrin light chain & SySy & $\begin{array}{l}113011 \\
\text { Monoclonal }\end{array}$ & $\sim 30$ & $\begin{array}{l}\text { Key component of the endocytic clathrin } \\
\text { coat }\end{array}$ \\
\hline Dynamin & SySy & $\begin{array}{l}115002 \\
\text { Polyclonal }\end{array}$ & $\sim 100$ & $\begin{array}{l}\text { GTPase involved in vesicle scission } \\
\text { during endocytosis }\end{array}$ \\
\hline $\begin{array}{l}\text { Glial fibrillary acidic } \\
\text { protein (GFAP) }\end{array}$ & AbD Serotec & $\begin{array}{l}46500280^{a} \\
\text { Polyclonal }\end{array}$ & $\sim 50$ & Glial cell marker \\
\hline Lactate dehydrogenase & Santa Cruz Biotechnology & $\begin{array}{l}\text { SC-33781 } \\
\text { Monoclonal }\end{array}$ & $\sim 35$ & Soluble protein \\
\hline Munc-18 & SySy & $\begin{array}{l}116011 \\
\text { Monoclonal }\end{array}$ & $\sim 66$ & $\begin{array}{l}\text { Membrane protein involved in vesicle } \\
\text { docking and fusion }\end{array}$ \\
\hline mUse1 & Custom Polyclonal27 & Not applicable & $\sim 31$ & $\begin{array}{l}\text { SNARE protein involved in ER-Golgi } \\
\text { transport }\end{array}$ \\
\hline Myelin basic protein & Dako & $\begin{array}{l}\text { A0623 } \\
\text { Monoclonal }\end{array}$ & $\sim 20$ & $\begin{array}{l}\text { Major protein component of the neuronal } \\
\text { myelin sheath }\end{array}$ \\
\hline $\mathrm{Na}^{+} / \mathrm{K}^{+}$-ATPase & Abcam & $\begin{array}{l}\text { AB7671 } \\
\text { Monoclonal }\end{array}$ & $\sim 110$ & $\begin{array}{l}\mathrm{Na}^{+} / \mathrm{K}^{+} \text {transporter specific for the } \\
\text { plasma membrane }\end{array}$ \\
\hline Rab3a & SySy & $\begin{array}{l}107111 \\
\text { Monoclonal }\end{array}$ & $\sim 25$ & Small GTPase associated with SVs \\
\hline Rab5 & SySy & $\begin{array}{l}108011 \\
\text { Monoclonal }\end{array}$ & $\sim 25$ & $\begin{array}{l}\text { Small GTPase associated with SVs and } \\
\text { recycling endosomes }\end{array}$ \\
\hline Rab-GDI & SySy & $\begin{array}{l}130011 \\
\text { Monoclonal }\end{array}$ & $\sim 65$ & Regulator of Rab protein activity \\
\hline Rpt 4 & Abcam & $\begin{array}{l}\text { AB22639 } \\
\text { Monoclonal }\end{array}$ & $\sim 44$ & Proteasome component \\
\hline $\begin{array}{l}\text { Succinate } \\
\text { dehydrogenase (SDHA) }\end{array}$ & Abcam & $\begin{array}{l}\text { AB14715 } \\
\text { Monoclonal }\end{array}$ & $\sim 70$ & Mitochondrial protein \\
\hline SNAP-25 & SySy & $\begin{array}{l}111111 \\
\text { Monoclonal }\end{array}$ & $\sim 25$ & $\begin{array}{l}\text { Plasma membrane SNARE protein } \\
\text { involved in neuronal exocytosis }\end{array}$ \\
\hline SNAP-47 & SySy & $\begin{array}{l}111403 \\
\text { Polyclonal }\end{array}$ & $\sim 50$ & $\begin{array}{l}\text { SNARE protein first identified on SVs. } \\
\text { Involved in LTP }\end{array}$ \\
\hline Spliceosome 61K & Custom Polyclonal28 & Not applicable & $\sim 55$ & Protein component of the spliceosome \\
\hline
\end{tabular}


PROTOCOL

TABLE 1 | Antibodies used in this study (continued).

\begin{tabular}{|c|c|c|c|c|}
\hline Synaptobrevin 2 & SySy & $\begin{array}{l}104211 \\
\text { Monoclonal }\end{array}$ & $\sim 18$ & $\begin{array}{l}\text { Vesicular SNARE protein involved in } \\
\text { neuronal exocytosis }\end{array}$ \\
\hline Synaptophysin 1 & SySy & $\begin{array}{l}101011 \\
\text { Monoclonal }\end{array}$ & $\sim 38$ & $\begin{array}{l}\text { Major SV membrane protein; involved in } \\
\text { endocytosis }\end{array}$ \\
\hline Synaptotagmin 1 & SySy & $\begin{array}{l}105011 \\
\text { Monoclonal }\end{array}$ & $\sim 60$ & $\mathrm{Ca}^{2+}$-sensor on SVs \\
\hline Syntaxin $1 \mathrm{~A}$ & SySy & $\begin{array}{l}110111 \\
\text { Monoclonal }\end{array}$ & $\sim 36$ & $\begin{array}{l}\text { Plasma membrane SNARE protein } \\
\text { involved in neuronal exocytosis }\end{array}$ \\
\hline
\end{tabular}

SySy, Synaptic Systems. Antibodies were used according to the supplier's recommendations.

aThis antibody has been discontinued. Suitable alternative antibodies/markers may be GFAP from SySy (cat. no. 173 011), or Aldh1L1 from Neuromab (cat. nos. 73-140 or 75-140) ref. 29.

\section{PROCEDURE}

I CAUTION Before starting, experimenters should ensure that animal experiments are carried out in accordance with relevant local and institutional animal protection regulations.

\section{Part 1: release of SVs from whole brain TIMING 45 min}

1) Decapitate one mouse (6-8 weeks postnatal, $25-30 \mathrm{~g}$ body weight, $0.5 \mathrm{~g}$ wet brain weight) and remove the whole brain. Wash the brain thoroughly with homogenization buffer.

$\triangle$ CRITICAL STEP The use of brains from older mice results in lower purity of the final SV fraction. This is probably due to the higher degree of myelination in older mice, with myelin-derived membrane fragments being a probable source of contamination.

$\triangle$ CRITICAL STEP After collecting the brains, all steps are carried out on ice or at $4{ }^{\circ} \mathrm{C}$.

2| Homogenize the brain in $9 \mathrm{ml}$ of ice-cold homogenization buffer (freshly supplemented with protease inhibitors, 0.001 volume), using a tight-fitting glass-Teflon homogenizer with ten strokes at 900 r.p.m.

$\triangle$ CRITICAL STEP As PMSF is unstable in aqueous solution, protease inhibitors should be added to the homogenization buffer only immediately before use. Stir the solution while adding protease inhibitors to avoid precipitation. Several vesicle proteins (e.g., synapsin I and synaptotagmin 1) are sensitive to proteolytic degradation by endogenous proteases.

\section{? TROUBLESHOOTING}

3| Centrifuge the homogenization mixture at 2,700 r.p.m. (1,000g) for $10 \mathrm{~min}$ at $4{ }^{\circ} \mathrm{C}$ in an SS34 rotor. Collect the supernatant (S1). The resulting pellet (P1), which contains large cell fragments and nuclei, can be discarded.

$\triangle$ CRITICAL STEP When collecting supernatants, do not disturb the pellets (including any material at the periphery;

Supplementary Fig. 1). Inclusion of pellet material results in higher contamination of the final vesicle fraction.

4| Centrifuge the S1 at 11,000 r.p.m. (15,000g) for $15 \mathrm{~min}$ at $4^{\circ} \mathrm{C}$ in an SS34 rotor. Collect the supernatant (S2) and store it on ice. The S2 contains SVs, along with small cell fragments, such as microsomes, small myelin fragments and also soluble proteins. The resulting pellet (P2) contains synaptosomes. Wash the pellet by carefully resuspending it in 1 ml of homogenization buffer (avoid the brown bottom part of the pellet, which consists mainly of mitochondria). Resuspension is best performed using a 1-ml pipette with the end cut off at the tip.

5| To release SVs from the synaptosomes, transfer the fraction into a tight-fitting glass-Teflon homogenizer; add 9 vol $(9 \mathrm{ml})$ of ice-cold $\mathrm{ddH}_{2} \mathrm{O}$ and perform three strokes at 2,000 r.p.m. Immediately add $50 \mu \mathrm{l}$ of $1 \mathrm{M} \mathrm{HEPES} \mathrm{(NaOH)} \mathrm{and} \mathrm{protease}$ inhibitors (0.001 volume).

$\Delta$ CRITICAL STEP Addition of protease inhibitors is critical at this stage because osmotic lysis of synaptosomes not only releases SVs but also neutral and lysosomal proteases into the solution. 


\section{Part 2: removal of contaminating proteins and membranes TIMING $3 \mathrm{~h} 45 \mathrm{~min}$}

6) Centrifuge the lysate at 12,000 r.p.m. $(17,000 \mathrm{~g})$ for $15 \mathrm{~min}$ at $4{ }^{\circ} \mathrm{C}$ in an SS34 rotor to remove all large membraneous particles (mostly mitochondria, junctional complexes and myelin). Collect the supernatant (LS1) and combine it with S2.

7| Centrifuge the LS1/S2 mixture at 20,000 r.p.m. $(48,000 \mathrm{~g})$ for $25 \mathrm{~min}$ at $4{ }^{\circ} \mathrm{C}$ in an SS34 rotor.

8| Collect the supernatant (CS1) and transfer it into a tight-fitting glass-Teflon homogenizer. SVs have a tendency to aggregate during the isolation procedure; homogenize the supernatant using 3-5 strokes at 900 r.p.m. To ensure proper disruption of any remaining SV clusters, draw the supernatant through a 20-gauge hypodermic needle attached to a 10-ml syringe, and then change to a 27-gauge needle and expel. Avoid the formation of air bubbles and foaming during this step. $\Delta$ CRITICAL STEP Proper disruption of clusters is essential to ensure uniform migration behavior in the subsequent centrifugation step.

9| Overlay $5 \mathrm{ml}$ of CS1 onto a $5-\mathrm{ml} 0.7 \mathrm{M}$ sucrose cushion. The number of tubes needed is dependent on the volume of CS1.

10| Centrifuge the sucrose cushion at 38,000 r.p.m. $(133,000 \mathrm{~g})$ for $1 \mathrm{~h}$ at $4{ }^{\circ} \mathrm{C}$ in a $70.1 \mathrm{Ti}$ rotor.

$\triangle$ CRITICAL STEP This rate-zonal centrifugation step separates SVs from protein particles, such as ribosomes and proteasomes, which otherwise co-sediment with SVs (see Supplementary Fig. 2 for more details on the centrifugation procedure). In our experience, proteasomes constitute a major contaminant that contributes substantially to the total protein in the final SV fraction, thereby lowering the effective enrichment levels of bona fide vesicle proteins.

11 After centrifugation, collect 500- $\mu$ fractions, starting from the top of the gradient. Fractionate the whole gradient. Also recover the white pellet that forms at the bottom of the tube by gently 'scraping' the tube wall with a plastic spatula, followed by transferring the pellet to a 1-ml homogenizer. Use a pipette tip with the end removed to reduce problems with pellet adherence to the plastic. Add $200 \mu \mathrm{l}$ of sucrose buffer and homogenize the mixture using three strokes at 900 r.p.m.

\section{Part 3: purity check by dot blotting TIMING $9 \mathrm{~h} 15$ min}

12| Spot $\sim 5 \mathrm{l}$ of each fraction (including the pellet) onto nitrocellulose paper and perform a dot blot assay (Fig. 2b; refs. 14,15). We include antibodies against an integral membrane protein on the SV (synaptophysin or synaptobrevin), as well as an antibody against a proteasome component (such as Rpt4).

13| SVs are usually found in fractions 12-20 (Fig. 2b). Combine these fractions with the pellet. Discard fractions 1-11. $\triangle$ CRITICAL STEP Discarding the fractions with substantial overlap between Rpt4 and synaptophysin (fractions 10 and 11 in Fig. 2b) markedly improves the purity of the final vesicle fractions at the cost of lower yield. If high yield is more important than purity, these fractions may be included.

\section{? TROUBLESHOOTING}

14| Collect the SVs into a pellet by ultracentrifugation (for example, by centrifuging the fractions at 50,000 r.p.m. $(300,000 \mathrm{~g})$ for $2 \mathrm{~h}$ in a $50.2 \mathrm{Ti}$ rotor). Although a $70.1 \mathrm{Ti}$ rotor can also be used at this stage, it uses low-capacity tubes. We prefer using the 50.2 Ti rotor, as the higher volume per tube results in the recovery of larger, easier-to-handle pellets.

\section{Part 4: size-exclusion chromatography as a final purification step $\bigcirc$ TIMING $1 \mathrm{~h}$, followed by overnight running of the size-exclusion column}

15| After centrifugation, discard the supernatant. Resuspend the pellet in $1 \mathrm{ml}$ of column buffer (SV-P); transfer the buffer to a 1-ml tight-fitting glass-Teflon homogenizer and perform ten strokes at 900 r.p.m. Draw the supernatant through a 20-gauge hypodermic needle attached to a 1-ml syringe; then change to a 27-gauge needle and expel. Again, avoid the formation of air bubbles and foaming during this step.

$\triangle$ CRITICAL STEP At this stage, it is estimated from electron micrographs that the vesicles are up to $20-30 \%$ contaminated by membranous fragments. Size-exclusion chromatography is omitted in many protocols but is essential in order to remove these remaining contaminating membranes and (if needed) remaining cytosolic proteins.

$\Delta$ CRITICAL STEP Two resins are available with a sufficiently large pore size that allows SVs to enter and, thus, to migrate behind the exclusion volume: controlled-pore glass beads ${ }^{10}$ (CPG-3000) and Sephacryl S-1000 (ref. 16). The former allows for robust columns that can be used for many years and can be operated at high flow rates because of the noncompressibility of the resin, resulting in substantially shortened separation times. Disadvantages include the low separation capacity (requiring sample:column ratios of 1:50 ( vol/vol)), limited commercial availability and high costs. Here we describe the use of Sephacryl S-1000, which gives acceptable levels of performance. The $100 \mathrm{~cm} \times 1 \mathrm{~cm}$ column used in this protocol can be used to 
purify SVs from one to four whole adult mouse brains, and the column size can be adjusted to accommodate smaller or larger samples from mice. It should be noted that, in our hands, one whole brain from an adult rat is equivalent to two brains from adult mice, on a wet weight:wet weight basis.

16 Load the SV-P onto an equilibrated Sephacryl S-1000 column and overlay it with column buffer. Connect the setup to a peristaltic pump and run it overnight with a flow rate of $\sim 6 \mathrm{ml} \mathrm{h}^{-1}$. Monitor the eluate by connecting a UV monitor to the bottom of the column (changes in signal at $280 \mathrm{~nm}$ will be due to a combination of aromatic amino acid absorbance by proteins and light scattering by larger particles). Collect the eluate coming through the UV monitor using a fraction collector. For best separation, individual fraction sizes of $0.7 \mathrm{ml}$ are recommended. SVs should have eluted from the column after 10 or $11 \mathrm{~h}$.

$\Delta$ CRITICAL STEP Avoid the temptation to use higher flow rates to speed up elution from the column. Sephacryl columns do not tolerate increased pressure. This results in compression of the material, which adversely affects resolution.

$\Delta$ CRITICAL STEP Sephacryl columns have a tendency to adsorb protein and membrane particles, in particular during the first few separation runs on a new column. Several vesicle preparations are needed to 'precondition' the Sephacryl column. Optimal yields are usually only obtained after the first three or four preparations. Preconditioning should be performed with SV-P. Avoid the temptation to use crude fractions, such as LP1, or alternatives such as BSA, which will ultimately affect vesicle purity.

$\triangle$ CRITICAL STEP Unlike CPG columns, the resolving capacity of Sephacryl columns depends on buffers containing salts with concentrations in the physiological range. Note that synapsins, which are peripheral vesicle proteins, dissociate from the vesicle membrane under these conditions ${ }^{11}$. Elution buffers of lower ionic strength (as required to preserve synapsin binding) substantially reduce resolution and increase contamination of the final SV preparation.

17| A successful column run produces two absorption maxima on the chromatogram (Fig. 3 ). The first peak contains larger membrane fragments (in the range of 100-200 nm in size). SVs (50 nm diameter) are found in the second peak. To obtain pure SVs, combine the fractions indicated in Figure 3; start from four to five fractions before the second maxima and continue combining fractions until just before the small bump in the trace (which represents elution of SVs with fractured membranes from the column). If purity can be sacrificed for yield, more fractions can be collected on either side of this second peak.

\section{Part 5: collection and storage of purified SVs TIMING 2 h 30 min}

18| Pool the desired fractions and centrifuge at 50,000 r.p.m. for $2 \mathrm{~h}$ at $4^{\circ} \mathrm{C}$ in a $50.2 \mathrm{Ti}$ rotor $(300,000 \mathrm{~g}$; see Step 14$)$. SVs should be visible as a clear, translucent pellet on the wall of the tube.

19| Discard the supernatant and resuspend the pellet (SV) in $\sim 200 \mu \mathrm{l}$ of the desired buffer. Transfer the buffer into a 1-mL tight-fitting glass-Teflon homogenizer and perform ten strokes at 900 r.p.m. Again, use a pipette tip with the end removed to reduce problems with pellet adherence to the plastic during transfer. Draw the supernatant through a 20-gauge hypodermic needle attached to a 1-ml syringe; then change to a 27 -gauge needle and expel, avoiding the formation of air bubbles and foaming.

\section{? TROUBLESHOOTING}

PAUSE POINT The purified SVs can be snap-frozen in liquid nitrogen and stored at $-80^{\circ} \mathrm{C}$.

Figure 3 | Effects of size-exclusion

chromatography on SV purity. (a) Elution profile of the Sephacryl column (monitored at $280 \mathrm{~nm}$ ). Roman numerals ( $\mathrm{i}-\mathrm{iii}$ ) on the chromatogram refer to fractions analyzed by electron microscopy (negative stain) shown in b. P1 and SV brackets indicate fractions pooled following elution from the column. (b) Negative-stain electron microscopy of fractions eluting from the size-exclusion column. The large, heterogeneous membrane structures present in the loading fraction ( $v$; SV-P-containing membranes with diameters of $100 \mathrm{~nm}$ or more) are concentrated in the first peak (P1) after chromatography (i). Homogenous small SVs with diameters ranging from 40 to $50 \mathrm{~nm}$ should be concentrated in the second peak (SV; iii). Fractions between these two peaks typically contain low amounts of membranes in both size ranges (ii). More than $90 \%$ of these small vesicles are immunopositive for the specific vesicle marker synaptophysin (iv; the inset shows immunogold-labeled vesicles at a higher magnification).

Scale bars in b: $500 \mathrm{~nm}$ (i-iii); $100 \mathrm{~nm}$ (iv, inset, v). a

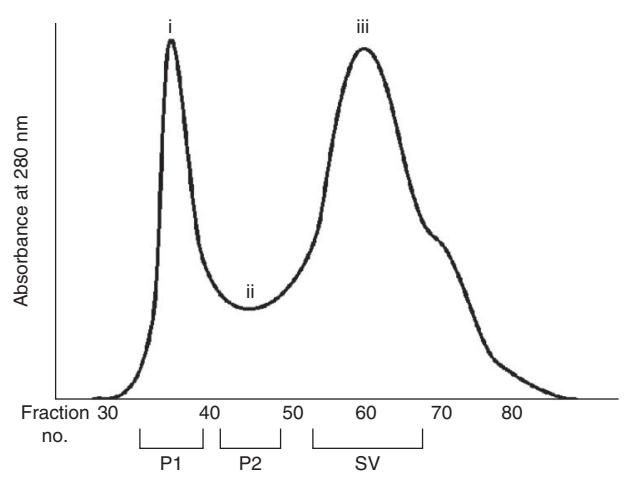

b
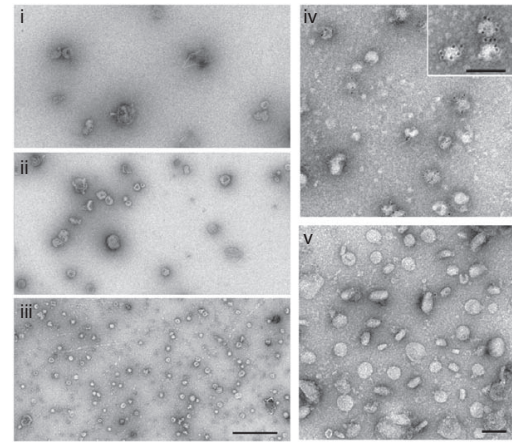


\section{? TROUBLESHOOTING}

Troubleshooting advice can be found in Table 2 .

TABLE 2 | Troubleshooting table.

\begin{tabular}{|c|c|c|c|}
\hline 2 & PMSF solubility & Use of ice-cold buffers & $\begin{array}{l}\text { PMSF is difficult to dissolve in ice-cold buffer. Add slowly } \\
\text { to solution, while stirring quickly. Expel the PMSF from the } \\
\text { pipette slowly and continuously. Do not remove the pipette } \\
\text { from the solution until the PMSF is fully added }\end{array}$ \\
\hline 13 & $\begin{array}{l}\text { Major contamination by } \\
\text { proteasomes (as judged by } \\
\text { Rpt4 immunoreactivity) }\end{array}$ & $\begin{array}{l}\text { Incorrect migration of SVs in } \\
\text { gradient during centrifugation }\end{array}$ & $\begin{array}{l}\text { SVs have a tendency to aggregate during the isolation } \\
\text { procedure. Increase homogenization of CS1 and pull/expel } \\
\text { through 20/27-gauge needles multiple times } \\
\text { Convex mixing of CS1 and sucrose cushion. Take care when } \\
\text { layering CS1 onto the cushion } \\
\text { Incorrect centrifugation conditions. Check rotors, speeds and } \\
\text { so on. Systematically vary centrifugation conditions until dot } \\
\text { blotting shows clear separation }\end{array}$ \\
\hline
\end{tabular}

Poor fractionation technique

19

\begin{abstract}
Poor vesicle yield
SV protein degradation (particularly synaptotag$\min 1$ )
\end{abstract}

Contamination (large membrane fragments)

Contamination (membrane fragments and proteins)
Too vigorous homogenization of whole brain

Poor resolution of size-exclusion column
Adsorption of membranes and proteins onto Sephacryl

Protease activity
Contamination (proteins; myelin)

Contamination (proteins; miscellaneous markers)
Completion of myelination relative to synapse formation is late

Poor fractionation technique

Incorrect pooling of fractions following size-exclusion chromatography
Gradients are fractionated from top to bottom. To ensure a clean fractionation, the pipette tip should be just in contact with the top of the gradient, not immersed under the surface. Fractions should be removed slowly

Run several vesicle preparations to 'pre-condition' Sephacryl column

Chill all buffers and centrifuge rotors before use. Keep samples on ice wherever possible, or at $4^{\circ} \mathrm{C}$

Check protease inhibitor activity. Prepare new stocks and add to the buffers immediately before use. Check whether PMSF has dissolved fully

Consider changing to Roche Complete tablets (EDTA-free), which are broad-spectrum protease inhibitor tablets and which retain stability in aqueous solution (Roche, cat. no. 1873 580)

Incorrect homogenizer clearance Try varying the initial homogenization conditions

Check individual fractions eluting from the size-exclusion column by negative-stain electron microscopy. Only pool fractions containing pure vesicles ( $40-50 \mathrm{~nm}$ diameter) Repack column. Generally longer columns give better separation

Avoid Sephacryl compression due to excessive column pressure. Check the flow rate

Avoid overloading the column with material Collect smaller volumes (shorten the collection time) for individual fractions

Use younger animals

Avoid cross-contamination of pellets and supernatants during the procedure

Dot blot individual fractions eluting from the size-exclusion column to identify fractions enriched in contaminating proteins and those enriched in SV markers 


\section{TIMING}

Steps 1-5, release of SVs from whole brain: $45 \mathrm{~min}$

Steps $6-11$, removal of contaminating proteins and membranes: $3 \mathrm{~h} 45 \mathrm{~min}$

Steps 12-14, purity check by dot blotting: 9 h 15 min

Steps $15-17$, size-exclusion chromatography as a final purification step: $1 \mathrm{~h}$, followed by overnight running of the

size-exclusion column

Steps 18 and 19, collection and storage of purified SVs: 2 h 30 min

\section{ANTICIPATED RESULTS}

The yield of SVs obtained using this preparation is $\sim 300 \mu \mathrm{g}$ of total protein per gram brain wet weight, which is approximately sixfold higher than that of the classical procedure using rat brain ${ }^{15}$ and more than two times higher than an alternative preparation claiming enhanced yield with comparable purity ${ }^{8}$. SVs that are purified and frozen remain structurally intact and functional (without loss of activity) for many months-as judged by the following criteria.

For studies of SV structure and function, it is essential to show that the vesicle fraction is free both from contamination by other membranes and particles produced as a by-product of the fractionation procedure and from associated cytoplasmic components. Traditionally, the methods used for assessing purity were largely confined to electron microscopic analysis of size and shape and to enzymatic assays to test for contaminating proteins originating from the mitochondria. Although electron microscopy remains a powerful tool, the identification of SV-specific proteins, such as synapsins, synaptotagmin and synaptophysin, means that biochemical homogeneity is now more easily assessed with immunological techniques such as immunoblotting - which allows SVs to be differentiated from other cellular transport vesicles or from vesiculated membrane fragments of similar size, which may have been generated during initial tissue homogenization.

In negative-stain electron micrographs, the final vesicle fraction should consist mainly of uniformly sized vesicles, with diameters ranging from 40 to $50 \mathrm{~nm}$. More than $90 \%$ of these vesicles should be immunoreactive for the integral membrane protein synaptophysin (Fig. $\mathbf{3 b}$ ). Furthermore, enrichment and yield, as well as the degree of contamination by other organelles and particles, can be monitored by immunoblotting fractions obtained during the purification for known

TABLE 3 | Quantification of SV enrichment during the isolation procedure.

\begin{tabular}{|c|c|c|c|c|c|c|}
\hline $\mathrm{H}$ & $4.8 \pm 0.5$ & $18.9 \pm 0.8$ & $90.1 \pm 12.3$ & $100.0 \pm 0.0$ & $100.0 \pm 0.0$ & $1.0 \pm 0.0$ \\
\hline $\mathrm{P} 1$ & $8.7 \pm 1.4$ & $2.6 \pm 0.4$ & $22.4 \pm 5.3$ & $25.0 \pm 4.4$ & $19.0 \pm 4.1$ & $0.8 \pm 0.1$ \\
\hline S1 & $3.7 \pm 0.6$ & $17.0 \pm 1.0$ & $63.3 \pm 10.5$ & $70.0 \pm 2.2$ & $63.0 \pm 6.7$ & $0.9 \pm 0.1$ \\
\hline S2 & $2.1 \pm 0.5$ & $15.3 \pm 1.5$ & $32.0 \pm 7.3$ & $35.0 \pm 3.0$ & $28.0 \pm 1.0$ & $0.8 \pm 0.1$ \\
\hline LP1 & $7.5 \pm 1.1$ & $1.7 \pm 0.6$ & $12.5 \pm 7.0$ & $14.0 \pm 5.3$ & $15.0 \pm 7.8$ & $1.1 \pm 0.2$ \\
\hline LS1 & $0.2 \pm 0.1$ & $15.5 \pm 3.9$ & $3.4 \pm 1.2$ & $4.0 \pm 1.7$ & $4.5 \pm 1.7$ & $1.1 \pm 0.3$ \\
\hline CS2P & $3.4 \pm 0.5$ & $0.1 \pm 0.1$ & $0.5 \pm 0.3$ & $0.5 \pm 0.4$ & $0.5 \pm 0.4$ & $1.0 \pm 0.0$ \\
\hline SV-P & $2.7 \pm 0.3$ & $0.7 \pm 0.1$ & $2.0 \pm 0.2$ & $2.2 \pm 0.2$ & $9.0 \pm 1.9$ & $4.1 \pm 0.2$ \\
\hline PI & $0.8 \pm 0.5$ & $0.1 \pm 0.1$ & $0.1 \pm 0.0$ & $0.1 \pm 0.1$ & $0.1 \pm 0.0$ & $0.7 \pm 0.1$ \\
\hline PII & $0.7 \pm 0.4$ & $0.1 \pm 0.0$ & $0.1 \pm 0.0$ & $0.1 \pm 0.0$ & $0.2 \pm 0.1$ & $1.7 \pm 0.5$ \\
\hline SV & $1.2 \pm 0.1$ & $0.2 \pm 0.0$ & $0.2 \pm 0.0$ & $0.3 \pm 0.1$ & $5.4 \pm 1.0$ & $20.0 \pm 1.0$ \\
\hline
\end{tabular}

The table shows the total protein recovery and relative enrichment of synaptophysin in the various subfractions taken during the isolation procedure, as assessed by quantitative immunoblotting. For synaptophysin, the enrichment in the final vesicle fraction should be 20 -fold over the starting homogenate. A similar level of enrichment should also be seen for other integral membrane proteins, such as synaptotagmin and synaptobrevin. Note the large amount of synaptophysin present in the S2 fraction, which would be lost in other protocols (Fig. 2). aProtein concentrations and volumes are given as indicators for a typical experiment. Protein concentration was measured using a modified Lowry procedure, with BSA as a protein standard. Figures represent the mean \pm s.d. from three independent experiments. Each experiment was performed using two mouse brains as the initial starting material. Figures are rounded to one decimal place. 
a

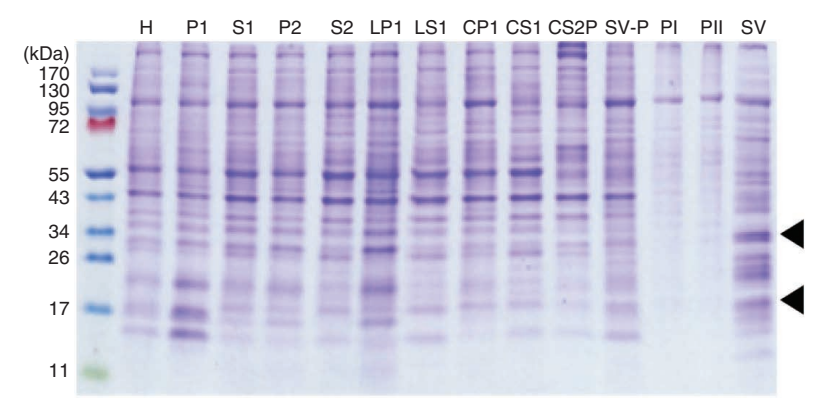

Figure 4 | Separation by SDS-PAGE of subfractions taken during the isolation of SVs. (a) Coomassie Blue staining. A volume of $5 \mu \mathrm{g}$ of total protein from each fraction was subjected to SDS-PAGE, followed by Coomassie staining. SVs (from mouse) have a characteristic protein profile. In a clean preparation, major protein bands at $18 \mathrm{kDa}$ and $38 \mathrm{kDa}$ should be clearly visible, which correspond to the major membrane proteins synaptobrevin and synaptophysin (arrowheads). (b) Immunoblots of the various subfractions. A volume of $5 \mu \mathrm{g}$ of total protein from each fraction was subjected to SDS-PAGE, followed by immunoblotting for various marker proteins. Known integral membrane proteins on SVs should enrich from homogenate to the final vesicle fraction. By contrast, proteins that are regarded as contaminants should be lost during the final purification steps. The dynamin antibody used does not discriminate between dynamin isoforms 1, 2 and 3, all of which are expressed in the brain. Note that to allow the most efficient use of membranes, they were cut into individual strips in the appropriate size range, as the specificity of all the antibodies used has been tested in previous publications (Table 1).

marker proteins. In general, markers for SVs, such as synaptophysin, synaptobrevin, vesicular neurotransmitter transporters and synaptotagmin, should all enrich in parallel, with the highest (typically 20-fold) enrichment being in the final SV fraction (Table 3 ). In contrast, markers for myelin and glia (MBP and GFAP), plasma membranes (for example, $\mathrm{Na}^{+} / \mathrm{K}^{+}$-ATPase and NMDA-R1) and mitochondria (SDHA) should be depleted (or at least not enriched) in the final fractions (Fig. 4 and Supplementary Fig. 3). The removal of protein super-complexes including proteasomes and spliceosomes (and perhaps ribosomes) proved to be the most difficult task, as these particles are similar to SVs in size. Rate-zonal gradient centrifugation turned out to be crucial to their removal, as these particles migrate more slowly than SVs, although their buoyant density is higher. Note that it is not possible to completely free the final SV fraction from transport vesicles that share similar physical properties, such as those involved in the traffic between the endoplasmic reticulum and the Golgi apparatus (mSec22 and muse1).

Under optimal conditions, the purity of our preparation compares well with that of the classical protocol developed by Whittaker and colleagues 6 , in which an extraordinary homogeneity can be obtained (ideally $99 \%$ purity; Huttner et al. ${ }^{11}$ ). With our preparation, the degree of contamination by other membranes is usually lower than $10 \%$, as judged both by morphology and immunolabeling. Furthermore, the enrichment of synaptophysin per mg of protein (20-fold) compares well with that of the classical protocol (23-fold; Table 3 and Jahn et al. ${ }^{15}$ ).

Purified SVs should also retain the ability to perform their primary in vivo functions-the ability to transport and store neurotransmitters and to fuse with the plasma membrane. Vesicular neurotransmitter uptake is mediated by specific transporters that depend on the proton electrochemical potential that exists across the vesicle membrane and which is generated by a vacuolar ATPase. Thus, vesicles purified using our procedure should show ATP-dependent glutamate uptake that is sensitive to the uncoupler carbonyl cyanide $p$-(trifluoromethoxy)phenylhydrazone (Fig. 5a). Failure to transport glutamate may well represent dissociation of the ATPase during purification (which is a problem associated with all 
Figure 5 | Testing SV function. (a) $\left[{ }^{3} \mathrm{H}\right] \mathrm{L}$-glutamate uptake by SVs isolated from mouse brain. SVs containing the vesicular glutamate transporter will accumulate glutamate under appropriate assay conditions. Glutamate uptake requires ATP-dependent vesicular acidification (produced by the vesicular proton pump). Addition of the uncoupler carbonyl cyanide $p$-(trifluoromethoxy) phenylhydrazone (FCCP) prevents glutamate uptake by dissipation of the proton gradient. Experiments were repeated five times for each condition, represented by the average uptake \pm s.d. (b) A fluorescent resonance energy transfer (FRET)-based lipid-mixing assay reports SNARE-mediated fusion of purified mouse SVs with liposomes. FRET occurs between the dyes NBD and Rhodamine, which are individually coupled to lipids used in the liposome preparation. FRET decreases (resulting in a measurable increase in NBD fluorescence) when vesicles fuse with liposomes containing the SNARE proteins syntaxin 1 and SNAP-25 (Syx/SN25 liposomes). This occurs as the larger (unlabeled) surface area of the fusion product allows greater spatial separation of the two dyes, resulting from lipid diffusion. In this figure, fusion is normalized to the total NBD fluorescence measured after liposomes are completely solubilized by Triton X-100, resulting in maximal spatial separation of NBD and Rhodamine. Fusion should be SNARE dependent. No fusion should be seen between SVs and empty liposomes, or when the vesicular SNARE protein synaptobrevin is cleaved with the light chain of tetanus toxin (TeNT). Fusion should also be effectively inhibited when liposomes and SVs are preincubated for $60 \mathrm{~min}$ at room temperature with the cytoplasmic fragments of either synaptobrevin or syntaxin/SNAP-25, respectively, as expected for competitive SNARE inhibition. Traces are representative of a typical experiment.

preparations $)^{17}$, or membrane damage that prevents the maintenance of a stable membrane potential. In vivo, fusion of SVs is mediated by SNARE (soluble NSF attachment protein receptor) proteins on the plasma membrane. Purified SVs should fuse with artificial membranes (liposomes) containing syntaxin 1 and SNAP-25 (Fig. 5b) ${ }^{18}$. Fusion should be SNARE dependent. No fusion should be seen between SVs and empty liposomes, or when the vesicular SNARE protein synaptobrevin is cleaved with the light chain of tetanus toxin. Fusion should also be effectively inhibited when liposomes and SVs are preincubated for $60 \mathrm{~min}$ at room temperature $\left(20^{\circ} \mathrm{C}\right)$ with the cytoplasmic fragments of either synaptobrevin or syntaxin/SNAP-25, respectively, as expected for competitive SNARE inhibition.

Detailed protocols for these assays - protein determination (Lowry method) and SDS-PAGE, Coomassie Brilliant Blue staining, immunoblotting, electron microscopy, glutamate uptake and vesicle fusion-have all been published previously3,8,18-21.

Anticipated uses Purified SVs were instrumental in identifying key proteins involved in exocytosis, such as synaptotagmin and synaptobrevin. Furthermore, purified SVs were the starting point for identifying novel families of membrane proteins that are probably involved in trafficking, including synaptophysins, synaptogyrins and SV2. More recently, SVs have served as a benchmark for comprehensive organelle proteomics ${ }^{2,3}$. Furthermore, purified SVs were used to develop the first quantitative molecular model of a trafficking organelle ${ }^{3}$. With the increase in sensitivity in many analytical tools, such as proteomics and fluorescence-based single-particle analysis, the ability to isolate SVs at high purity from small samples such as single mouse brains opens a new window for comparative biochemistry, involving both structural and functional approaches. In particular, the vesicle preparation described here will be very useful in the characterization of the increasing variety of transgenic mouse lines that contain targeted alterations of vesicular and other synaptic components. Furthermore, vesicles isolated from mice carrying GFP-tagged SV proteins will provide novel tools for refined in vitro analysis, which so far has not been possible. Notably, our preliminary experiments also suggest that the use of transgenic animals will not be limited to those that survive to adulthood; experiments aimed at 'downscaling' the protocol still further indicate that it will be possible to eventually purify SVs from embryonic brains for analysis.

Many in vitro assays are available for the study of various aspects of SV function. The substantially increased yield achieved by our protocol will both increase experimental throughput and reduce the number of animals that need to be killed for a given experiment. In addition to neurotransmitter uptake and in vitro reconstitution of SNARE-mediated membrane fusion, isolated SVs can be used to study, for instance, the function of the vacuolar ATPase 8,22 , to monitor binding and unbinding of Rab-GTPases 23 , to measure dissociation of other peripheral proteins such as synapsins ${ }^{11}$, to map the topology of membrane proteins 24,25 and to study the function of the calcium sensor synaptotagmin 26 .

Note: Supplementary information is available in the online version of the paper.

ACKNOWLEDGMENTS We thank M. Druminski for excellent technical assistance during the project. E. Reisinger and P. D'Adamo provided synaptobrevin 1-GFP knock-in mice and GDI knockout mice, respectively, which were used in 'proofof-principle' experiments during protocol development. I. Herford prepared rat hippocampal cultures. G. van den Bogaart and H. Martens (Synaptic Systems) gave much useful advice. S.A. is a student of the Gauss PhD program of the Georg-August University, Göttingen. This work was funded by the Max Planck
Society (R.J.) and by a European Research Council Starting Grant (AstroFunc: 281961) (M.H.).

AUTHOR CONTRIBUTIONS The experimental work was performed and analyzed by S.A. and M.H., with the exception of the electron microscopy, which was performed by D.R. The project was conceived and supervised by R.J., in conjunction with M.H. S.A., M.H. and R.J. wrote the manuscript.

COMPETING FINANCIAL INTERESTS The authors declare no competing financial interests. 
Reprints and permissions information is available online at http://www.nature. com/reprints/index.html.

1. Jahn, R. \& Südhof, T.C. Membrane fusion and exocytosis. Annu. Rev. Biochem. 68, 863-911 (1999).

2. Morciano, M. et al. Immunoisolation of two synaptic vesicle pools from synaptosomes: a proteomics analysis. J. Neurochem. 95, 1732-1745 (2005).

3. Takamori, S. et al. Molecular anatomy of a trafficking organelle. Cell 127, 831-846 (2006).

4. De Robertis, E., Rodriguez De Lores Arnaiz, G., Salganicoff, L., Pellegrino De Iraldi, A. \& Zieher, L.M. Isolation of synaptic vesicles and structural organization of the acetycholine system within brain nerve endings. J. Neurochem. 10, 225-235 (1963).

5. Whittaker, V.P., Michaelson, I.A. \& Kirkland, R.J. The separation of synaptic vesicles from nerve-ending particles ('synaptosomes'). Biochem. J. 90, 293-303 (1964).

6. Whittaker, V.P. The synaptic vesicle. in Handbook of Neurochemistry, Vol. 7: Structural Elements of the Nervous System (ed. Lajtha, A.) Ch. 2, 41-69 (Plenum Press, 1984).

7. Gray, E.G. \& Whittaker, V.P. The isolation of nerve endings from brain: an electron-microscopic study of cell fragments derived by homogenization and centrifugation. J. Anat. 96, 79-88 (1962).

8. Hell, J.W., Maycox, P.R., Stadler, H. \& Jahn, R. Uptake of GABA by rat brain synaptic vesicles isolated by a new procedure. EMBO J. 7 , 3023-3029 (1988).

9. Park, Y. et al. Controlling synaptotagmin activity by electrostatic screening. Nat. Struct. Mol. Biol. 19, 991-997 (2012).

10. Nagy, A., Baker, R.R., Morris, S.J. \& Whittaker, V.P. The preparation and characterization of synaptic vesicles of high purity. Brain Res. 109, 285-309 (1976).

11. Huttner, W.B., Schiebler, W., Greengard, P. \& De Camilli, P. Synapsin I (protein I), a nerve terminal-specific phosphoprotein. III. Its association with synaptic vesicles studied in a highly purified synaptic vesicle preparation. J. Cell Biol. 96, 1374-1388 (1983).

12. Hu, K. et al. Vesicular restriction of synaptobrevin suggests a role for calcium in membrane fusion. Nature 415, 646-650 (2002).

13. Burger, P.M. et al. Synaptic vesicles immunoisolated from rat cerebral cortex contain high levels of glutamate. Neuron 3, 715-720 (1989).

14. Jahn, R., Schiebler, W. \& Greengard, P. A quantitative dot-immunobinding assay for proteins using nitrocellulose membrane filters. Proc. Natl. Acad. Sci. USA 81, 1684-1687 (1984).
15. Jahn, R., Schiebler, W., Ouimet, C. \& Greengard, P. A 38,000-Dalton membrane protein (p38) present in synaptic vesicles. Proc. Natl. Acad. Sci. USA 82, 4137-4141 (1985)

16. Stadler, H. \& Tsukita, S. Synaptic vesicles contain an ATP-dependent proton pump and show 'knob-like' protrusions on their surface. EMBO J. 3, 3333-3337 (1984).

17. Moriyama, Y. \& Nelson, N. Cold inactivation of vacuolar proton-ATPases. J. Biol. Chem. 264, 3577-3582 (1989).

18. Holt, M., Riedel, D., Stein, A., Schuette, C. \& Jahn, R. Synaptic vesicles are constitutively active fusion machines that function independently of $\mathrm{Ca}^{2+}$. Curr. Biol. 18, 715-722 (2008).

19. Harlow, E. \& Lane, D. Antibodies: A Laboratory Manual. (Cold Spring Harbor Laboratory Press, 1999).

20. Takamori, S., Rhee, J.S., Rosenmund, C. \& Jahn, R. Identification of a vesicular glutamate transporter that defines a glutamatergic phenotype in neurons. Nature 407, 189-194 (2000).

21. Takamori, S., Riedel, D. \& Jahn, R. Immunoisolation of GABA-specific synaptic vesicles defines a functionally distinct subset of synaptic vesicles. J. Neurosci. 20, 4904-4911 (2000).

22. Maycox, P.R., Deckwerth, T., Hell, J.W. \& Jahn, R. Glutamate uptake by brain synaptic vesicles. Energy dependence of transport and functional reconstitution in proteoliposomes. J. Biol. Chem. 263, 15423-15428 (1988).

23. Chou, J.H. \& Jahn, R. Binding of Rab3A to synaptic vesicles. J. Biol. Chem. 275, 9433-9440 (2000).

24. Perin, M.S., Brose, N., Jahn, R. \& Südhof, T.C. Domain structure of synaptotagmin (p65). J. Biol. Chem. 266, 623-629 (1991).

25. Stenius, K., Janz, R., Südhof, T.C. \& Jahn, R. Structure of synaptogyrin (p29) defines novel synaptic vesicle protein. J. Cell Biol. 131, 1801-1809 (1995).

26. Brose, N., Petrenko, A.G., Südhof, T.C. \& Jahn, R. Synaptotagmin: a calcium sensor on the synaptic vesicle surface. Science 256, 1021-1025 (1992).

27. Verrier, S.E. et al. Members of a mammalian SNARE complex interact in the endoplasmic reticulum in vivo and are found in COPI vesicles. Eur. J. Cell Biol. 87, 863-878 (2008).

28. Makarova, O.V., Makarov, E.M., Liu, S., Vornlocher, H.P. \& Lührmann, R. Protein $61 \mathrm{~K}$, encoded by a gene (PRPF31) linked to autosomal dominant retinitis pigmentosa, is required for U4/U6* U5 tri-snRNP formation and pre-mRNA splicing. EMBO J. 21, 1148-1157 (2002).

29. Barres, B.A. The mystery and magic of glia: a perspective on their roles in health and disease. Neuron 60, 430-440 (2008). 